\title{
An Evolutionary Algorithm for QoS Routing under Uncertain Parameters
}

\author{
Fangguo He Huan Qi Qiong Fan \\ Institute of Systems Engineering, Huazhong University of Science \& Technology, Wuhan 430074, P. R. China
}

\begin{abstract}
In the actual network the information collected through routing protocols is uncertain with the expansion of network size and complexity. In this paper, the QOS routing problem is investigated in the networks environment with uncertain parameters. The fuzzy set theory is used to define the relationship between two fuzzy numbers, and construct a QOS routing model of multi-objective optimization. A multi-objective evolutionary algorithm (MOEA) is utilized to solve the problem. Simulation shows the MOEA can provide an available approach to QoS routing in networks environment with uncertain parameters.
\end{abstract}

Keywords: Fuzzy set, QOS routing, Evolutionary algorithm, Multi- objective optimization

\section{Introduction}

With the increasing demand in real-time application such as internet telephony, video conferencing and other multimedia applications, an efficient allocation of network resources satisfying quality of service (QoS) requirements is becoming the primary goal of routing problem. The QoS requirements of a connection include a wide range of parameters such as bandwidth, delay, delay jitter and loss ratio. As known, the QoS routing under multiple constraints is a NPcomplete problem, and is difficult to get a polynomialtime solution [1], Recently, Several QoS routing algorithms, such as heuristic algorithms and approximation algorithms, have been proposed [2]-[3], most of these algorithms depend on accurate information about the topology and availability of resources in networks. However, in the actual network the information collected through routing protocols is uncertain with the expansion of network size and complexity. The main causes of uncertainty information in communication networks are due to unavailable parameters, inaccurate calculations and aggregated information in hierarchical networks [4]. Therefore, in reality, the uncertainty of network state information cannot be ignored, and should be properly treated and expressed so that the QoS constraints are satisfied.

At present, much research attention has been focused on QoS routing problem under the uncertain network state information [4]-[6]. Guerin and Orda investigated the problem of QoS routing when the state information is uncertain or inaccurate and expressed through probabilistic model [5]. They presented a network framework with uncertain parameters, and focused on the QoS routing problem for bandwidth and delay demands. Korach and Ohayon [6] studied the problem of path selection when the information available for making the decision is inaccurate. Cohen et al. proposed a fuzzybased method for path selection under the inaccurate information for making routing decisions [4]. So far, most of the work in QoS routing of uncertain state information is till in the theoretical development stage, and the proposed algorithm mainly based on probabilistic model. In fact, fuzzy number is a kind of effective approach to express uncertain parameter in the uncertain system. It is proved that the fuzzy set theory can provide a convenient and powerful framework for dealing with information characterized by imprecision and uncertainty. So, in this work, we draw upon concepts from fuzzy set theory to describe the imprecision and uncertainties in the QOS routing problem.

\section{Preliminaries}

The concept of fuzzy set was initiated by Zadeh via membership function in 1965 . The fuzzy set theory has been successfully applied in many field including industry, agriculture, medicine and society. In this section we give some definitions and theorems needed in this research.

Definition 1: A fuzzy set $A$ defined on $R=(-\infty, \infty)$ which has the following membership function is called a standard fuzzy number

$$
u_{A}(x)= \begin{cases}0, & |x-a|>\Delta a \\ 1-(x-a) / \Delta a, & |x-a| \leq \Delta a\end{cases}
$$


By the definition, a standard fuzzy number $A$ is determined by the 2-tuple $(a, \Delta a)$ with $\Delta a<a$, whose membership function is shown in Fig.1. We denote a standard fuzzy number $A$ in $A=(a, \Delta a)$, where $a$ is the center, and $\Delta a$ is the width. In our study, the fuzzy number means the standard fuzzy number because of its widely application.

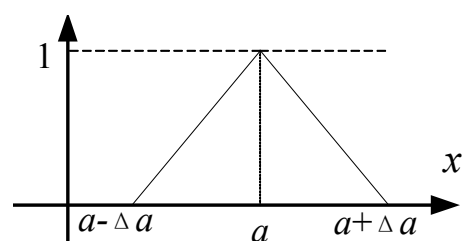

Fig.1: The fuzzy number $A$

Theorem1: Suppose that $A=(a, \Delta a)$ and $B=(b$, $\Delta b)$ are fuzzy numbers, then the reliability of $A<B$ is given by the following [7]

$$
\operatorname{Re} l(A<B)=\left\{\begin{array}{cc}
1, & b-a \geq \Delta a+\Delta b \\
\frac{1}{2}\left(1-\frac{a-b}{\Delta a+\Delta b}\right), & |b-a|<\Delta a+\Delta b \\
0, & b-a \leq-(\Delta a+\Delta b)
\end{array}\right.
$$

Where Rel denotes reliability, which is defined the degree of the fuzzy number $A$ smaller than the fuzzy number $B$. In order to treat the problem conveniently, we use $R(A, B)$ denote $\operatorname{Rel}(A<B)$, i.e. $R(A, B)=\operatorname{Rel}(A<B)$. According to the theorem, in particular, when $B$ is accurate number $b$, i.e. $\Delta b=0$, the possibility of $A<b$ is denoted by

$$
\mathrm{R}(A, b)= \begin{cases}1, & b-a \geq \Delta a \\ \frac{1}{2}\left(1-\frac{a-b}{\Delta a}\right), & |b-a|<\Delta a \\ 0, & b-a \leq-\Delta a\end{cases}
$$

It is easy to verify that $R(A, B)$ has the property: $R(A, B)+R(B, A)=1$.The theorem 1 is the basis of theory to deal with QOS routing problem applying fuzzy number. In order to describe the sum of fuzzy number, we have the following theorem.

Theorem2 [8]: Let $A_{i}=\left(a_{i}, \Delta a_{i}\right)$ be fuzzy number, where $i \in\{1,2 \ldots, n\}$, then the equation holds.

$$
\sum_{i=1}^{n} A_{i}=\left(\sum_{i=1}^{n} a_{i}, \sum_{i=1}^{n} \Delta a_{i}\right)
$$

\section{Network Model}

A network is usually represented by a weighted digraph $G=(V, E)$, where $V$ denotes the set of nodes and $E$ denotes the set of communication links. Without loss of generality, we only considered the digraphs in which there exists at most one link between a pair of nodes. For any link $l \in E$, a group of fuzzy parameter, written as $\left(D_{l}, B_{l}, C_{l}\right)$, is associated with it, where the fuzzy number $D_{l}=\left(d_{l}, \Delta d_{l}\right), B_{l}=\left(w_{l}, \Delta w_{l}\right)$ and $C_{l}=$ $\left(c_{l}, \Delta c_{l}\right)$ denote the delay, bandwidth and cost of link $l$, respectively. By the theorem 2, the relationship between the individual link delay and the path delay denoted by $D_{p}$ can represent as follows

$$
D_{p}=\left(d_{p}, \Delta d_{p}\right)=\sum_{l \in p} D_{l}=\left(\sum_{l \in p} d_{l}, \sum_{l \in p} \Delta d_{l}\right)
$$

Similarly, the relationship between the individual link cost and the path cost denoted by $C_{p}$ can represent as follows

$$
C_{p}=\left(c_{p}, \Delta c_{p}\right)=\sum_{l \in p} C_{l}=\left(\sum_{l \in p} c_{l}, \sum_{l \in p} \Delta c_{l}\right)
$$

Obviously, $D_{p}$ and $C_{p}$ are also fuzzy number, which we will utilize to construct a fuzzy model of QOS routing. In order to explicit the following problem clearly, we first give an illustrative example to explain the essence of the problem.

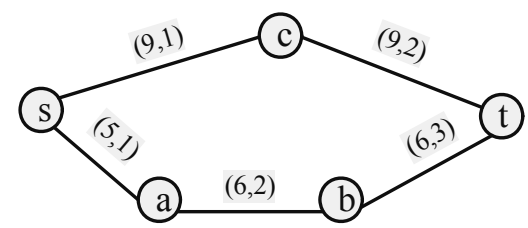

Fig.2: Graph of the fuzzy number

Fig. 2 shows a simple graph where two possible paths exist from a source node $s$ to a destination node $t$. The fuzzy number associated with each link represents delay, and the constraint of end-to-end delay is $b=20$. If only search the optimal solution according to the center value, the path $s-a-b-t$ is selected. While in terms of reliability, according to the formulation (3), the optimal solution is the path s- $\mathrm{c}-\mathrm{t}$, since its reliability is bigger than the other $(5 / 6>3 / 4)$.

Consider a QoS routing request $R=(S, T, d, b, c)$, where $S$ and $T$ are the source node and the end node respectively, $d, b$ and $c$ are all accurate number and represent bandwidth requirement, delay requirement and cost requirement, respectively. Our goal is for each QoS request $R$ to construct a routing path $p$ satisfying the following properties:

1) The reliability that the delay of the path $p$ is smaller than the constraint $d$ should be maximal, i.e.

$$
\max f_{1}(p)=R\left(D_{p}, d\right)
$$

2) The reliability that the cost of the path $p$ is smaller than the constraint $c$ should be maximal, i.e.

$$
\max f_{2}(p)=R\left(C_{p}, c\right)
$$

3) The multiplication of the reliability that the bandwidth of the link $l$ is bigger than the constraint $b$ should be maximal, i.e.

$$
\max f_{3}(p)=\prod_{l \in p} p\left(b, B_{l}\right)
$$


Thus, the QoS routing problem satisfying the QoS request $R$ can be formulated into a mathematic optimization. As known, the optimization problem is a multi-objective optimization, which can't be solved by simply method.

\section{The utilized algorithm}

Multi-objective optimization addresses problems with several design objectives, which are often conflicting. There exists no best solution to the problem, but a set of compromise solutions. The set of compromise solutions is referred to as the non-dominated or Pareto set of solutions.

Definition 2: For a maximum problem $F(x)=$ $\left(f_{1}(x) \ldots f_{k}(x)\right)$, a decision vector $u$ is said to dominate a decision vector $v$ (also written as $u \succ v$ ) if and only if: $\forall i \in\{1, \ldots, k\}, f_{i}(u) \geq f_{i}(v)$ and $\exists i \in\{1, \ldots, k\}: f_{i}$ $(u)>f_{i}(v)$.

Definition 3: A solution $x^{*}$ is said to be Pareto optimal or a efficient solution if and only if there is no $x$ such that $f_{i}(x) \geq f_{i}\left(x^{*}\right)$ for all $i \in\{1,2, \ldots, k\}$, with at least one strict inequality. Pareto optimal solution is also called non-dominated solution, and the set of all non-dominated objective vectors is called the Pareto front $(\mathrm{PF})$.

\subsection{Fitness value and niche count}

In this research, a Pareto optimal solution means a Pareto optimal path. Our algorithm will look for the set of non-dominated paths that will satisfy the three different QoS parameters, namely end-to-end delay, bandwidth and cost guarantee. We will use a multiobjective evolutionary algorithm (MOEA) to solve the problem without combining them into a single objective function.

Comparative studies have shown that, among all major evolutionary algorithms, the strength Pareto evolutionary algorithm proposed by Zitzler \& Thiele is clearly superior [9]. Aiming at the problem of this research, we improved properly the algorithm so as to deal with the multi-objective shortest path. The detailed implementation of the algorithm is as follows:

Step 1: Generate random initial population $P$ and create the empty external set of non-dominated individuals $P^{\prime}$.

Step2: Evaluate the three sub-objective function for each individual in $P$ in parallel.

Step 3: Copy non-dominated members of $P$ to $P^{\prime}$.

Step 4: Remove solutions within $P^{\prime}$ which are dominated by any other member of $P^{\prime}$.

Step 5: If the number of externally stored nondominated solutions exceeds a given maximum $N^{\prime}$, prune $P^{\prime}$ by means of crowding distance.
Step 6: Calculate the fitness of each individual in $P$ as well as in $P^{\prime}$.

Step 7: Select individuals from $P+P^{\prime}$, until the mating pool is filled.

Step 8: Perform crossover and mutation to members of the mating pool in order to create a new population $P$.

Step 9: If maximum number of generations is reached, then stop, else go to Step 2.

\subsection{Encoding and initial population}

For the reason that the model in this study is to solve the end-to-end problem, we adopt a direct encoding method, which a chromosome represents a path from the source node to the end node. For example, a path consisting of nodes $1,5,11$ and 14 can be coded as a string $(1,5,11,14)$.

At the beginning of initialization, the source node (gene) is considered as the current node. Then, the second node (gene) is chosen at random from the successor of current node. Later, the chosen node is regarded as the current node and the procedure repeats itself until the current node can be connected to the end node directly. In order to escape from loop during generating path, the method is adopted to make a mark on the chosen node, which ensures that this node will not be visited again.

\subsection{The process of evolution}

(1) Fitness assignment and evolution Selection In Step 6, all individuals in $P$ and $P^{\prime}$ are assigned a scalar fitness value. This is accomplished in the following two-stage process.

Step 1: Each solution $i \in P^{\prime}$ is assigned a value $s_{i} \in[0,1]$, called strength, $s_{i}$ is defined as $s_{i}=\frac{m}{n+1}$.

Where $m$ denotes the number of individuals in $P$ that are dominated by $i$ and $n$ is the size of $P$. The fitness $f_{i}$ of $i$ is equal to its strength: $f_{i}=s_{i}$

Step 2: The fittness of an individual $j \in P$ is calculated by summing the strengths of all external non-dominated solutions $i \in P^{\prime}$ that dominated $j$. Add one to this sum to guarantee that members of $P^{\prime}$ always have better fitness than members of $P$ (the fitness is to be minimized): $f_{i}=1+\sum_{i, i \succ j} s_{i}$

In Step 7, selection was done using the binary tournament procedure: Randomly select two individuals out of the population $P$, then copy the one with the better fitness value to the mating pool until the mating pool is full.

(2) Crossover and mutation 
Two chromosomes can be crossed if they have at least one pair of same gene (node) except for source and destination nodes. If there are more pairs of common genes, one pair is randomly chosen and the locus of each node becomes a crossing site of each chromosome. An example of the crossover procedure is given as follows:

parent1: | 1235669 | parent2: | 13346789 |

offspring1: | 12346789 | offspring2: | 13569 |

In order to perform a mutation, a node, called mutation point, is randomly selected from the chosen path. Then, another node is chosen randomly from the node's successor, repeats the procedure until a node can be connected to the end node directly. It is noticed that nodes already included in an upper partial path should be marked so as not to include the same node twice in the new path

\subsection{Reduction by crowding distance}

In Step 5, the number of externally stored nondominated solutions is limited to some number $N^{\prime}$. We adopt the method of crowding distance to provite the external set from growing to infinity. The crowding distance is calculated as below:

(1) Sort the individuals of $P^{\prime}$ based on objective $m$, i.e. $I=\operatorname{sort}(m)$.

(2) Assign infinite distance to boundary values for each individual, i.e. $I\left(d_{1}\right)=\infty$ and $I\left(d_{\mathrm{n}}\right)=\infty$

(3) for $k=2$ to $(n-1)$

$$
I\left(d_{k}\right)=I\left(d_{k}\right)+\frac{I(k+1) \cdot m-I(k-1) \cdot m}{f_{m}^{\max }-f_{m}^{\min }}
$$

Where $I(k) . m$ is the value of the $m^{\text {th }}$ objective function of the $k^{\text {th }}$ individual in $I$. The individual of larger distance is chosen to remain. Obviously, the individuals in the boundary are always selected since they have infinite distance assignment

\section{Simulation}

In the section, the performance of the algorithm was tested on the basis of Waxman's model [10] with 50 nodes. The probability that there exists edge between any two nodes is expressed by

$$
p(u, v)=\alpha \exp (-d(u, v) / \beta D)
$$

where $d(u, v)$ is geometric distance from node $u$ to node $v, D$ is maximum distance between two nodes, parameter $\beta$ determines the scale of short edge and long edge of the random graph, and parameter $\alpha$ determines the average degree of the random graph.

We utilize the proposed algorithm to simulate the fuzzy model, which were performed with MATLAB.
Assumed that the delay $D_{l}=\left(d_{l}, \Delta d_{l}\right)$, bandwidth $B_{l}=$ $\left(w_{l}, \Delta w_{l}\right)$ and cost $C_{l}=\left(c_{l}, \Delta c_{l}\right)$ of link $l$ are all randomly generated, where $d_{l}, \Delta d_{l}, w_{l}, \Delta w_{l}, c_{l}$ and $\Delta c_{l}$ is uniformly distributed in the range of $[10,50 \mathrm{~ms}],[0$, $5 \mathrm{~ms}],[40,100 \mathrm{Mb} / \mathrm{s}],[0,10 \mathrm{Mb} / \mathrm{s}],[10,40]$ and $[0,4]$, respectively. The delay and cost constraint of application are assumed to respectively distribute in $[40,150 \mathrm{~ms}]$ and $[50,200]$ while bandwidth requirement is variable.

We used our algorithm to compare the exhaustive search method, which finds the optimal values of the three QoS parameters by exhaustively searching them one after another. Fig. 3 shows that how these Paretooptimal fronts are developed and converged towards a global-optimal solution, and the table 1 explains the ratio of Pareto paths during evolution.
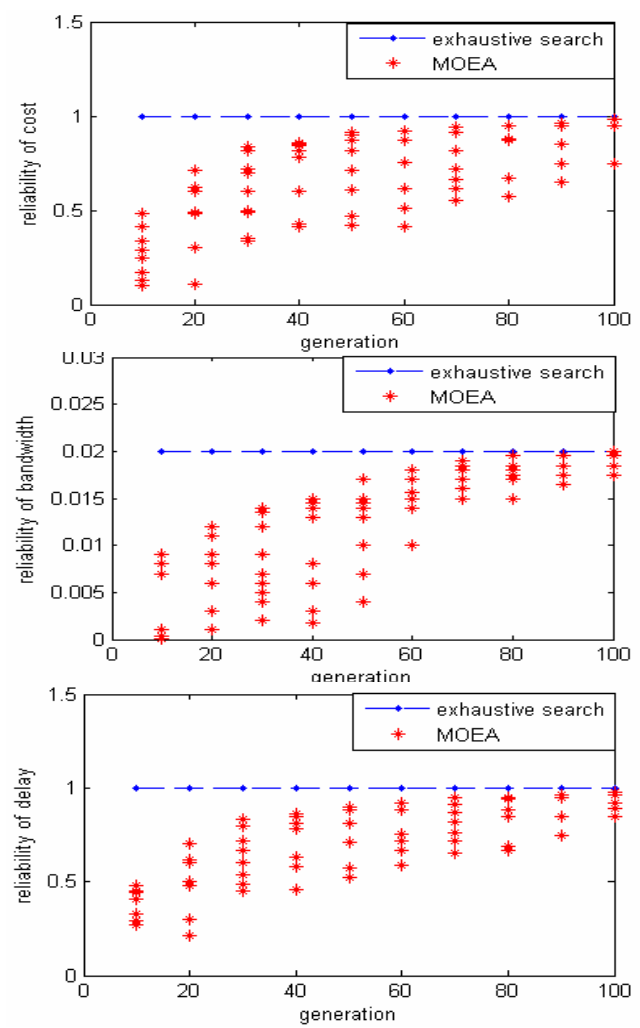

Fig. 3: Evolutionary process

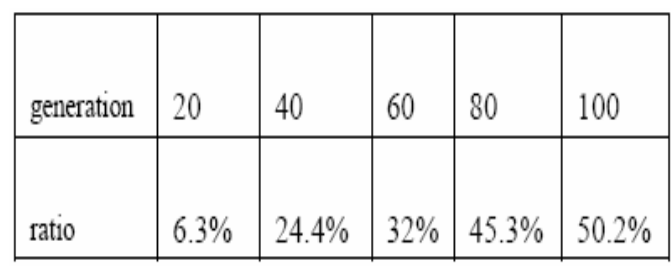

Table 1: The ratio of parato paths.

\section{Conclusions}


In this research, we investigated the QOS routing problem in the networks environment with uncertain parameters. We utilize the fuzzy set theory to define the relationship between two fuzzy numbers, and construct a QOS routing model of multi-objective optimization according to the theory of reliability. The multi-objective evolutionary algorithm (MOEA) is utilized to solve the problem. Simulation experiments demonstrate the availability and efficiency of MOEA. Our study shows that MOEA can provide an available approach to QoS routing in networks environment with uncertain parameters.

\section{References}

[1] Z. Wang, J. Crowcroft, Quality of Service for Supporting Multimedia application. IEEE, Journal on Selected Areas in Communication, 14(7):1228-1234, 1996.

[2] H.De Neve, and P.Van Mieghem, TAMCRA a tunable accuracy multiple constraints routing algorithm. Computer Communications, 23(7): 667-679, 2000.

[3] Turgay Korkmaz, and Marwan Krunz, MultiConstrained Optimal Path Selection. Proc. of IEEE Infocom'2001 (Anchorage, Alaska, USA), 1.2: 834-843, 2001.

[4] A. Cohen, E. Korach, M. Last and R. Ohayon, Afuzzy-based path ordering algorithm for QoS routing in non-deterministic communication networks. Fuzzy Sets and Systems, 150: 401-417, 2005.

[5] R.A. Guerin and A.Orda, QoS routing in networks with inaccurate information: theory and algorithms. IEEE/ACM Transactions on Networking, 6(6): 350-364, 1999.

[6] D.H.Lorenz and A.Orda, QoS routing in networks with uncertain parameters. IEEE/ACM Transactions on Networking, 6(12): 350-364, 1998.

[7] Zhang Pin, Li Le-min and Wang Sheng, Using fuzzy number to solve routing problems on the uncertain condition. ACTA ELECTRONICA SINICA, 31(12):1861-1865, 2003.

[8] H. Tanaka and K. Asai, Fuzzy solution in fuzzy linear programming problems. IEEE Trans on System, Man and Cybernetics, 14:325-328, 1984.

[9] E. Zitzler and L. Thiele, Multiobjective Evolutionary Algorithms: A Comparative Case Study and the Strength Pareto Approach. IEEE Transactions on Evolutionary Computation, 3(4): 257-271, 1999.

[10] B.M. Waxman, Routing of multipoint connections. IEEE Journal of Selected Area in Communications, pp. 1617-1622, 1998. 